\title{
Electrochemical Conversion of Copper-Based Hierarchical Micro/ Nanostructures to Copper Metal Nanoparticles and Their Testing in Nitrate Sensing
}

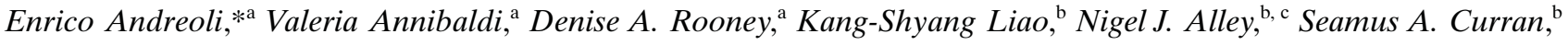 \\ Carmel B. Breslin \\ a Department of Chemistry, National University of Ireland Maynooth, Maynooth, Co. Kildare, Ireland \\ b Institute for NanoEnergy, University of Houston, 3575 Cullen Boulevard, 617 Science and Research I, Building 550, Houston, TX \\ $77204-5005$ \\ c School of Physical Sciences, Dublin City University, Glasnevin, Dublin 9, Ireland \\ *e-mail: chemistry.department@nuim.ie
}

Received: February 24, 2011

Accepted: June 6, 2011

\begin{abstract}
Metallic copper nanoparticles (CuNP) are formed from the reduction of hierarchical copper-based micro/nanostructures previously electrodeposited on polypyrrole-polystyrene sulfonate (PPy-PSS) thin films. The application of a high reduction potential causes the erosion of the micro/nanostructures nanosheets and the formation of CuNP. The CuNP are used in a series of preliminary tests in order to assess their performance in the sensing of nitrate. The sensing performance is improved with the introduction of polyethyleneimine-functionalized multiwall carbon nanotubes (MWNT-PEI) to the PPy-PSS films. Electrochemical impedance spectroscopy studies show that the nanotubes increased the electronic conductivity of the reduced films. The limit of detection of the PPy-PSS-MWNT-PEI-CuNP nanocomposite is $30 \mu \mathrm{M}$ nitrate. The materials need further development and optimization work in order to be applied as sensors.
\end{abstract}

Keywords: Polypyrrole, Copper nanoparticles, Multiwall carbon nanotubes, Nitrate sensing

DOI: 10.1002/elan.201100105

\section{Introduction}

Nanomaterials have been studied and used in sensors and molecular switches because of their unique scaling and sensitivity-to-change properties [1-3]. In particular there has been intense interest in the use of nanoparticles in electroanalytical applications $[4,5]$. Copper nanoparticles have been applied for the electrochemical sensing of many different analytes like, for example, carbohydrates, amino acids, nitric oxide, nitrite and nitrate [6]. Nitrate, in particular, is hazardous for both the environment, causing eutrophication of natural water when in the presence of phosphates, and human health, leading to infant methemoglobinemia from nitrate dietary intake $[7,8]$.

Nitrate can be detected by several spectroscopic, chromatographic and electrochemical methods [9]. Electrochemical methods are attractive since they are usually simpler to apply. Copper nanoparticles and copper modified electrodes have been used for the electrochemical sensing of nitrate $[6,10,11]$, and detection limits as low as $0.76 \mu \mathrm{M}$ have been achieved [10]. The limit set by the EU Drinking Water Directive is $50 \mathrm{ppm}$ in nitrate content corresponding to about $0.8 \mathrm{mM}$ nitrate [12], whereas the US Environmental Protection Agency set a limit of
$10 \mathrm{ppm}$ in nitrogen content corresponding to about $0.7 \mathrm{mM}$ nitrate [13].

Copper nanomaterials have been electrodeposited on polypyrrole films using different methodologies. The deposition of copper nanocrystals on polypyrrole ultrathin films allows precise control of the distribution and morphology of the deposit through the variation of polymer thickness, electrode potential and electrolyte concentration [14]. The type of deposition, galvanostatic or potentiostatic, also influences the characteristics of the copper nanodeposits $[15,16]$. The deposition of metal nanoparticles on conducting polymers is also affected by the initial oxidation state of the polymer, the presence of metal/ polymer interactions, and by the use of metal ion complexes instead of free metal ions [17].

The present manuscript is focused on the preparation, characterization and preliminary testing of novel nanocomposite materials comprising polypyrrole, carbon nanotubes and copper metal nanoparticles. Copper-based hierarchical micro/nanostructures attached to a PPy-PSS film are converted to CuNP upon application of a high reduction potential. The structures crumble into particles under the combined action of direct reduction and hydrogen gas evolution. The method has certain advantages over other 
approaches as it is simple to apply and allows the synthesis of large amounts of independent CuNP.

The CuNP formed on the PPy-PSS film are tested for nitrate sensing applications. A drawback of having nanoparticles supported on conducting polymer films is the insulating barrier introduced by the polymer at reduction potentials. This is true in sensing applications, where the electrons required to reduce the analyte have to cross the resistive film in order to be supplied to the nanoparticles. A solution to this problem is to introduce conducting pathways in the film by incorporating carbon nanotubes (CNT) added as a composite or in a functionalized form depending on the needs in terms of conductance $[18,19]$. The dispersion of CNT in water requires either functionalization or the addition of dispersing agents $[20,21]$. In particular, functionalizing nanotubes is beneficial when more monodispersed layers are required [22]. High conductivities are achieved when multiwall carbon nanotubes (MWNT) are dispersed in polymer hosts. However, they can also diminish the continuance of the film to specific pathways if they are allowed to aggregate [23,24]. Our findings show that the sensing performance of the PPyPSS-CuNP film is enhanced by introducing polyethylenimine-functionalized multiwall carbon nanotubes (MWNT-PEI) in the polymer film. The novel materials presented in this manuscript show promising nitrate sensing properties, although their applicability as a sensing device requires to be further checked experimentally.

\section{Experimental}

\subsection{Chemicals and Instrumentation}

The chemicals were pyrrole (98\%), poly(sodium 4-styrenesulfonate) $M_{\mathrm{w}} \approx 70000 \mathrm{~g} / \mathrm{mol}$, copper sulfate pentahydrate $(99.999 \%)$, sodium sulfate $(\geq 99.0 \%)$, polyethylenimine branched $M_{\mathrm{w}} \approx 25000 \mathrm{~g} / \mathrm{mol}$ (impurities $\leq 1 \%$ ), dimethylformamide $(\geq 99.0 \%)$, methanol $(\geq 99.0 \%)$ and acetone ( $\geq 99.5)$ supplied by Sigma-Aldrich, and multiwall carbon nanotubes $10-30 \mathrm{~nm}$ diameter and $0.5-40 \mu \mathrm{m}$ length $(95 \%)$ supplied by Helix Material Solutions. Oxygen gas $(99.95 \%)$ was supplied by BOC Ireland. Pyrrole was vacuum-distilled and stored in the dark at $-20^{\circ} \mathrm{C}$ under nitrogen. Water was distilled and Milli-Q purified.

Electrochemical experiments were performed in air or oxygen-saturated solutions at room temperature in a standard three-electrode configuration cell [25] connected to either a potentiostat Solartron 1285 or a Solartron 1287 coupled to a Solartron 1260A. The rotating disk electrode (RDE) was a Pine MRS with E3 tips. All potentials are referred to saturated calomel electrode (SCE).

Scanning electron microscopy was performed with a Hitachi S-3200-N equipped with Oxford Instrument INCAx-act, and a FEI Nova NanoSEM 630 ultra-high resolution equipped with Oxford Instrument X-MAX 80.

\subsection{Preparation of Materials}

\subsubsection{Synthesis of MWNT-PEI}

Multiwall carbon nanotubes (MWNT) modified with polyethylenimine (PEI) were synthesized as reported by Liao et al. [26]: $1.0 \mathrm{~g}$ of MWNT and 5.6 g of PEI were placed in a stoppered round-bottomed flask $(250 \mathrm{~mL})$ with $60 \mathrm{~mL}$ of dimethylformamide. The mixture was sonicated for $10 \mathrm{~min}$ and stirred at $50^{\circ} \mathrm{C}$ for $5 \mathrm{~d}$. $150 \mathrm{~mL}$ of methanol was added to the reaction mixture which was left stirring and cooling for $1 \mathrm{~h}$. The mixture was vacuum-filtered on a Whatman Teflon filter with $0.45 \mu \mathrm{m}$ pores. The black paste was washed repeatedly with aliquots of methanol (total $100 \mathrm{~mL}$ ) and transferred to a clean round-bottomed flask $(250 \mathrm{~mL})$ to which $100 \mathrm{~mL}$ of methanol was added. After sonication, the dispersion was stirred at room temperature for $2 \mathrm{~h}$ and filtered again. The paste was further washed with $250 \mathrm{~mL}$ of Milli-Q water, with $10 \mathrm{~mL}$ of acetone and left drying for $30 \mathrm{~min}$. The black chunks were finely grounded and dried overnight at $60^{\circ} \mathrm{C}$ on a bed of silica gel beads.

\subsubsection{Preparation of MWNT-PEI Aqueous Solution}

$60 \mathrm{mg}$ of the MWNT-PEI powder was added to $10 \mathrm{~mL}$ of Milli-Q water in a glass vial. The suspension was sonicated for $1 \mathrm{~h}$ and left stirring at room temperature for $24 \mathrm{~h}$. The solution was centrifuged at $14000 \mathrm{rpm}$ for $10 \mathrm{~min}$. The deep black supernatant was collected with a Pasteur pipette. The amount of nanotubes in solution was estimated by removing the water from $5 \mathrm{~mL}$ of stock solution and obtaining approximately $4 \mathrm{mg}$ of residue, corresponding to about $0.08 \% \mathrm{w} / \mathrm{w}$ of MWNT-PEI in water. No carbon nanotube precipitation was observed over a period of a year.

\subsubsection{Preparation of PPy-PSS-MWNT-PEI Films}

PPy-PSS films were deposited on bare electrodes from a solution $0.15 \mathrm{M}$ pyrrole and $50 \mathrm{mM}$ PSS at $0.60 \mathrm{~V}$ vs. SCE until a charge of $40 \mathrm{mC} / \mathrm{cm}^{2}$ was reached [25]. PPy-PSSMWNT-PEI films were prepared as follows. A glassy carbon (GC) electrode was pretreated to make the surface hydrophilic and allow a smooth distribution of the nanotubes [27-29]. The electrode (4 $\mathrm{mm}$ diameter) was placed in a stirred phosphate buffer solution $(0.20 \mathrm{M}$ $\mathrm{NaH}_{2} \mathrm{PO}_{4} / \mathrm{Na}_{2} \mathrm{HPO}_{4}, \mathrm{pH} 6.8$ ) and held at $1.80 \mathrm{~V}$ vs. SCE for $5 \mathrm{~min}$. Immediately after, a CV scan was performed from 0.30 to $1.25 \mathrm{~V}$ vs. SCE at $25 \mathrm{mV} / \mathrm{s}$ until a reproducible cyclic voltammogram was obtained (about 30 cycles). The electrode was washed with Milli-Q water and acetone. After drying, the surface acquired a blue iridescent colour. A $5 \mu \mathrm{L}$ drop of MWNT-PEI solution was cast onto the electrode surface and dried under an infrared lamp. At this stage, the electrode surface became dull and grey. A PPy-PSS film was electrodeposited onto the carbon nanotube layer as described at the beginning of this section for the bare electrodes. 


\subsubsection{Preparation and Conversion of Copper-Based Hierarchical Structures to Copper Nanoparticles}

Copper-based hierarchical micro/nanostructures were electrodeposited as described elsewhere [25]. Briefly, the flower-like structures were formed on PPy-PSS thin films upon application of a potential of $0.10 \mathrm{~V}$ vs. SCE in aerated or oxygen-saturated $0.10 \mathrm{M} \mathrm{CuSO}_{4}$. These were converted to metallic copper nanoparticles by applying a potential of $-2.00 \mathrm{~V}$ vs. SCE for $5 \mathrm{~min}$ in $0.10 \mathrm{M} \mathrm{Na}_{2} \mathrm{SO}_{4}$ Solution. A large amount of hydrogen gas was generated at the newly formed copper nanoparticles, the composite film was undamaged by the gas evolution. After reduction, the electrode surface was characterized by a bright and shiny pinkish colour.

\subsubsection{Nitrate Sensing with Copper Nanoparticles Modified Electrodes}

The copper nanoparticles were formed on PPy-PSS and PPy-PSS-MWNT-PEI films. The modified electrodes were immersed in a $0.10 \mathrm{M} \mathrm{Na}_{2} \mathrm{SO}_{4}$ solution. The RDE was set $-1.20 \mathrm{~V}$ vs. SCE and at $2000 \mathrm{rpm}$. A series of nitrate aliquots of $1-100 \mu \mathrm{M}$ were added into the solution. Sensing tests were also performed using cyclic voltammetry. The modified electrodes were scanned in $0.10 \mathrm{M}$ $\mathrm{Na}_{2} \mathrm{SO}_{4}$ from -0.60 to $-1.50 \mathrm{~V}$ vs. $\mathrm{SCE}$ at $10 \mathrm{mV} / \mathrm{s}$. The nitrate aliquots ranged from 0.1 to $10.0 \mathrm{mM}$.

\section{Results and Discussion}

\subsection{Conversion of Copper-Based Hierarchical Micro/ Nanostructures to Metallic Copper Nanoparticles}

\subsubsection{Formation of CuNP onto PPy-PSS Films}

The electrochemical deposition and characterization of copper-based hierarchical micro/nanostructures have been discussed in detail previously [25]. The structures were made of copper sulfate hydroxide hydrate, $\left[\mathrm{CuSO}_{4}\right]_{2}\left[\mathrm{Cu}(\mathrm{OH})_{2}\right]_{3} \cdot 5 \mathrm{H}_{2} \mathrm{O}$, and posnjakite, $\mathrm{CuSO}_{4^{-}}$ $\left[\mathrm{Cu}(\mathrm{OH})_{2}\right]_{3} \cdot \mathrm{H}_{2} \mathrm{O}$.

The morphological changes of the hierarchical micro/ nanostructures upon the application of $-2.00 \mathrm{~V}$ vs. SCE in $0.10 \mathrm{M} \mathrm{NaSO}_{4}$, for two different time intervals, are shown in Figure 1. After the application of this potential for $1 \mathrm{~min}$, the hierarchical structures (Figure 1a) become surrounded by a uniform and sparse distribution of nanoparticles (Figure 1b). If the potential is held for $5 \mathrm{~min}$, the hierarchical structures disappear and in their place circular distributions of CuNP are found (Figure 1c).

A closer inspection of the micro/nanostructure reveals the actual erosion of the nanosheets due to the reduction, from their smooth appearance (1d) in the pristine state to the jagged borders (1e) after reduction. The erosion of the hierarchical structure is probably due to the evolution of hydrogen gas at the newly formed CuNP. The larger CuNP are found at the original site of the clusters, while the smaller are distributed in the surrounding areas. Interestingly, the large CuNP are distributed in an O-ring shape (Figure 1c). The micrograph at high magnification (1f) shows the presence of two main size ranges. The
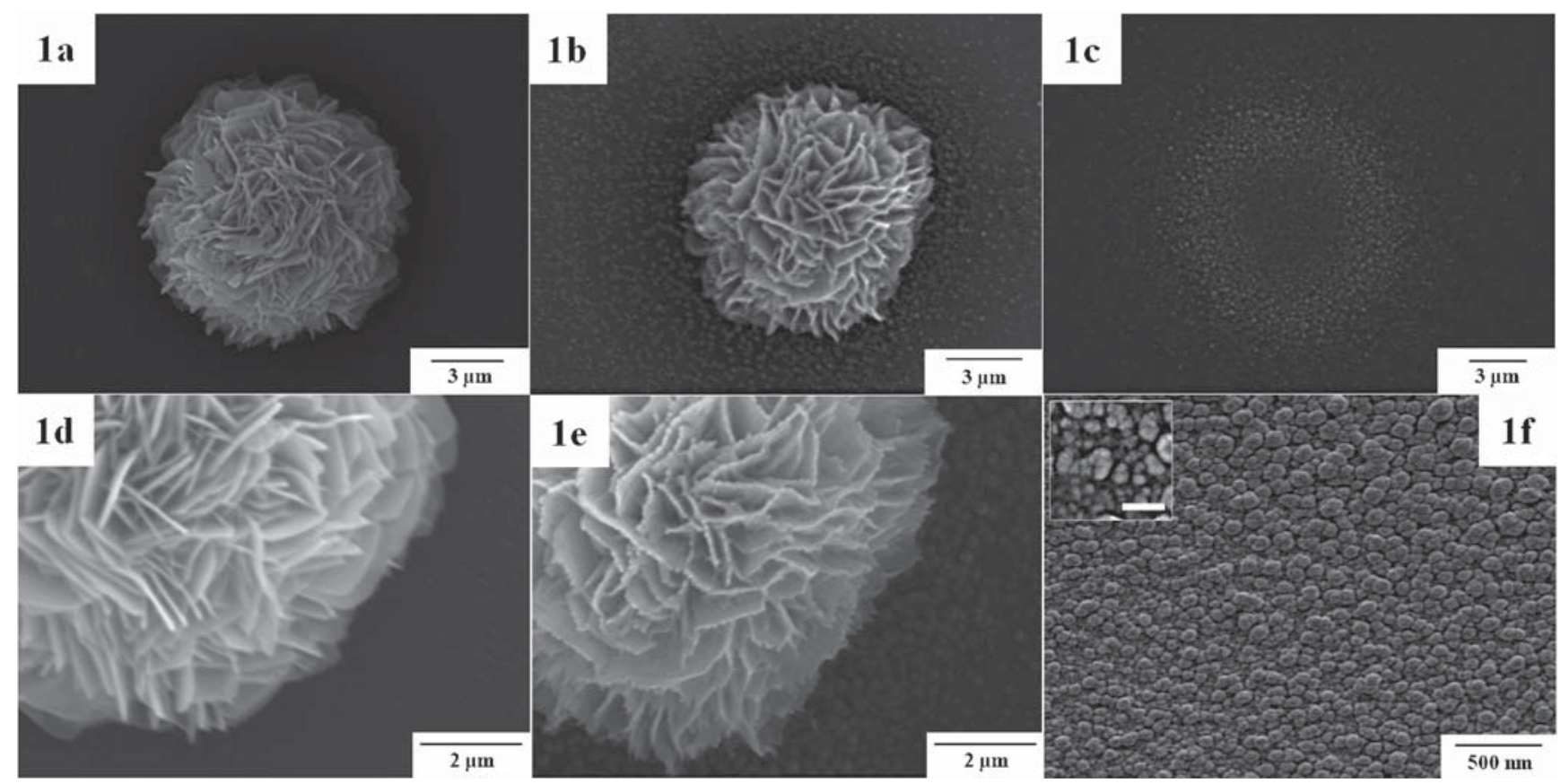

Fig. 1. Hierarchical copper-based micro/nanostructures before and after reduction. The pristine structure in (a) and (d) is reduced in $0.10 \mathrm{M} \mathrm{Na}_{2} \mathrm{SO}_{4}$ at $-2.00 \mathrm{~V}$ vs. SCE for $1 \mathrm{~min}$ to give the eroded structure in (b) and (e), or at $-2.00 \mathrm{~V}$ vs. SCE for $5 \mathrm{~min}$ to give the distribution of CuNP shown in (c). (f) High resolution micrograph of the CuNP. Inset: scale bar $=100 \mathrm{~nm}$. 


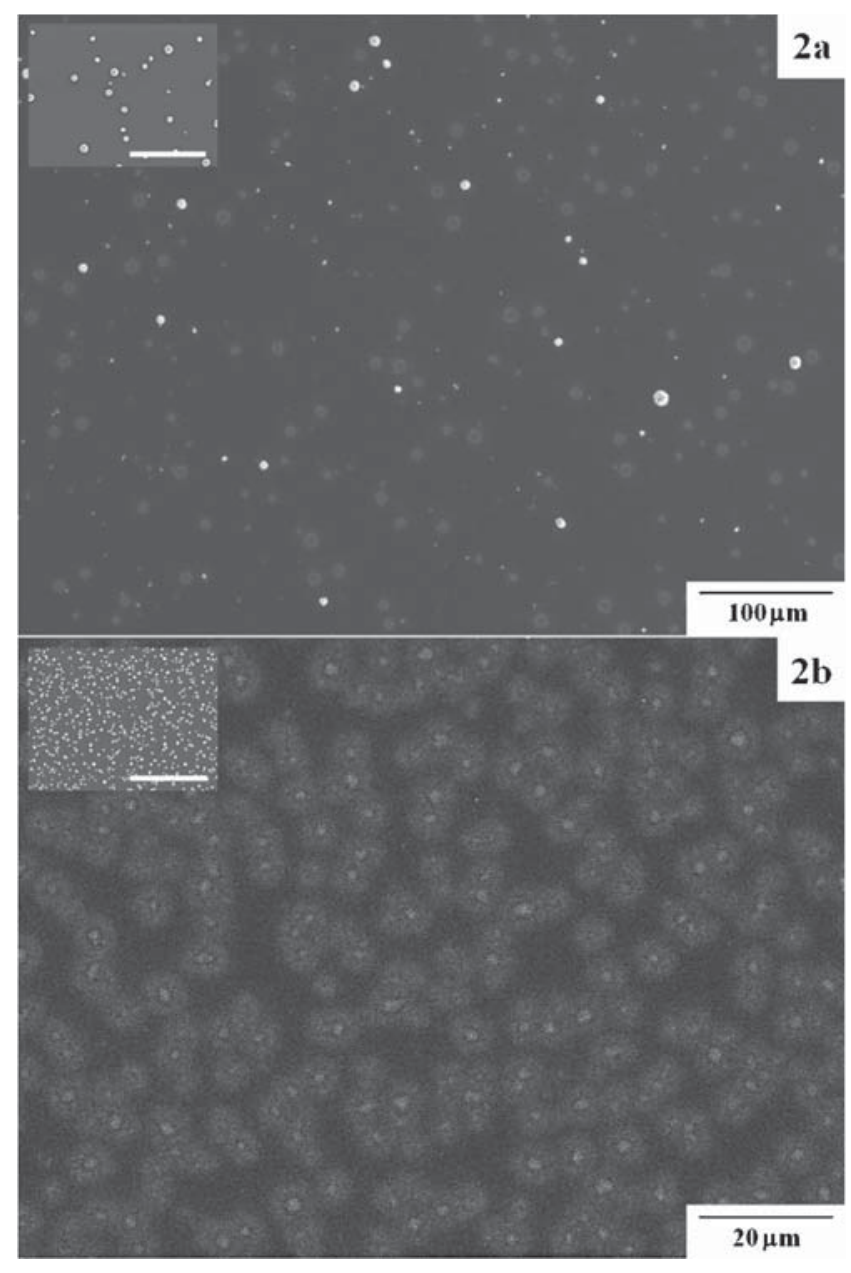

Fig. 2. CuNP produced from the reduction of hierarchical copper-based micro/nanostructures at $-2.00 \mathrm{~V}$ vs. SCE in $0.10 \mathrm{M}$ $\mathrm{Na}_{2} \mathrm{SO}_{4}$. In a), CuNP formed from clusters deposited in aerated $0.10 \mathrm{M} \mathrm{CuSO}_{4}$ solutions; in b), CuNP formed from clusters deposited in oxygen-saturated solutions (in the insets the scale bars are $60 \mu \mathrm{m})$.

larger CuNP range from 50 to $100 \mathrm{~nm}$, while the smallest ones are $10 \mathrm{~nm}$ (inset). The distribution of CuNP on the film is directly related to the original position of the clusters, as shown in Figure 2. Clusters deposited in air are few and large (Figure 2a) giving a sparse distribution of CuNP, instead those deposited from oxygen-saturated solutions are numerous and small (Figure $2 \mathrm{~b}$ ) giving a dense distribution.

\subsubsection{Preparation of CuNP onto PPy-PSS-MWNT-PEI Films}

It is known that the incorporation of CNT inside conducting polymers can increase the conductivity and capacitance of PPy films [30-32]. MWNT-PEI are soluble in water and can be easily drop-cast on an anodized glassy carbon surface. The resulting layer of MWNT-PEI is shown in Figure 3. The surface is homogenously covered by entangled MWNT-PEI. The diameter of the larger nanotubes is around $50 \mathrm{~nm}$ compared to the $30 \mathrm{~nm}$ of the pristine MWNT. This corresponds to an approximate thickness of about $10 \mathrm{~nm}$ for the PEI functionalization. The layer is very porous with some voids visible even at lower magnification. The weight of MWNT-PEI per unit surface is estimated to be about $32 \mu \mathrm{g} / \mathrm{cm}^{2}$ based on the diameter of the electrode $(4 \mathrm{~mm})$, and the volume and concentration of the drop-casting solution $(5 \mu \mathrm{L}$ and $0.08 \% \mathrm{w} / \mathrm{w}$, respectively).

After polymerization of pyrrole in the presence of the polyanionic dopant, PSS, a novel nanocomposite film of PPy-PSS-MWNT-PEI was formed. The nanocomposite film is marked by large cracks (Figure 3b) which are not observed for the PPy-PSS film prepared under similar experimental conditions (Figure 3c). In addition to this microporosity, some mesoporosity is also likely to be present, as observed in comparable PPy films prepared from MWNT-PSS dispersions [33].

The hierarchical copper-based micro/nanostructures were successfully prepared and reduced to CuNP on the PPy-PSS-MWNT-PEI films, as shown in Figure 4. The hierarchical structures were deposited from oxygen-saturated solutions under the same conditions used for PPyPSS films [25]. The CuNP are distributed on the top of the nanocomposite film as previously seen for the PPyPSS films. The larger CuNP are observed on the site of the original structures, while the smaller CuNP are distributed in the neighbouring areas.

\subsection{Nitrate Sensing}

CuNP are known for their catalytic activity towards the reduction of nitrate ions. Accordingly, the PPy-PSS films with and without MWNT-PEI and modified with CuNP were tested as nitrate sensors. The mechanism of reduction of nitrate is still in debate, but the main products formed are ammonia and hydroxylamine [34]. Nitrite ions can also be formed at copper electrodes [35].

\subsubsection{PPy-PSS-CuNP Electrode}

The nitrate sensing performance of a PPy-PSS-CuNP modified electrode was tested using cyclic voltammetry. Representative data are shown in Figure 5a. The modified electrode was cycled in the presence of different concentrations of nitrate, ranging from $0.1 \mathrm{mM}$ to $10.0 \mathrm{mM}$. The current response increases with increasing nitrate concentration. This increase is not observed at PPy-PSS films; hence it is due to the catalytic activity of the CuNP. The broad reduction wave is typical of slow reduction processes, under kinetic controlled conditions. The wave can be related to the reduction of nitrates and/or further reduction of the products of nitrate reduction, e.g., nitrite ions. At the highest concentration $(10.0 \mathrm{mM})$ two very small signals emerge from the wave at about $-0.90 \mathrm{~V}$ and $-1.10 \mathrm{~V}$ vs. SCE. In order to prepare a calibration curve, the current recorded at $-1.20 \mathrm{~V}$ vs. SCE is plotted against the nitrate concentration (with previous subtraction of the background current registered without nitrate in solu- 

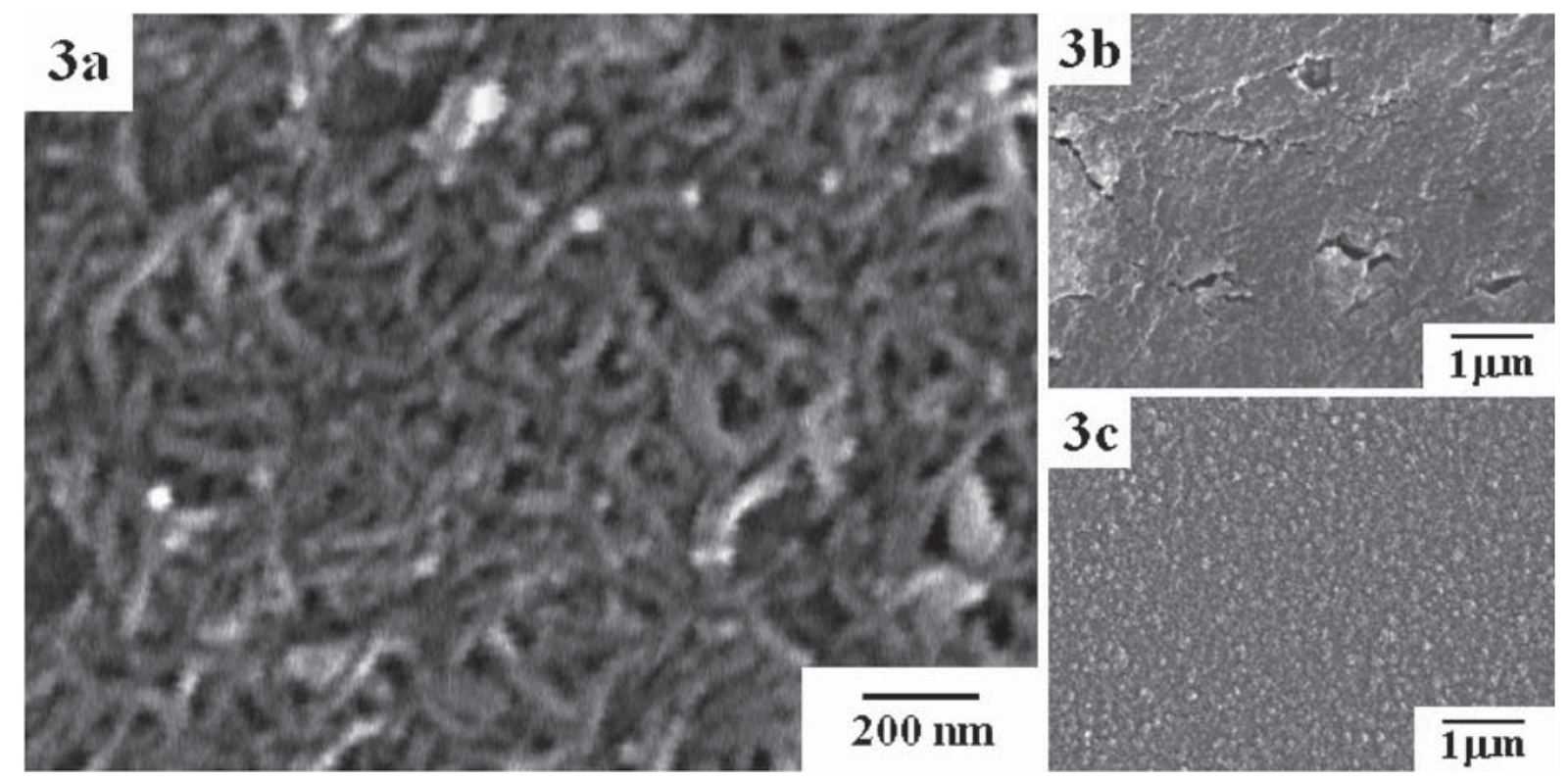

Fig. 3. Electrode surface modified with MWNT-PEI before and after the deposition of the PPy-PSS film. a) Carbon nanotubes homogeneously distributed on the surface. b) PPy-PSS film grown on the MWNT-PEI-modified electrode; c) PPy-PSS film formed on the bare electrode.
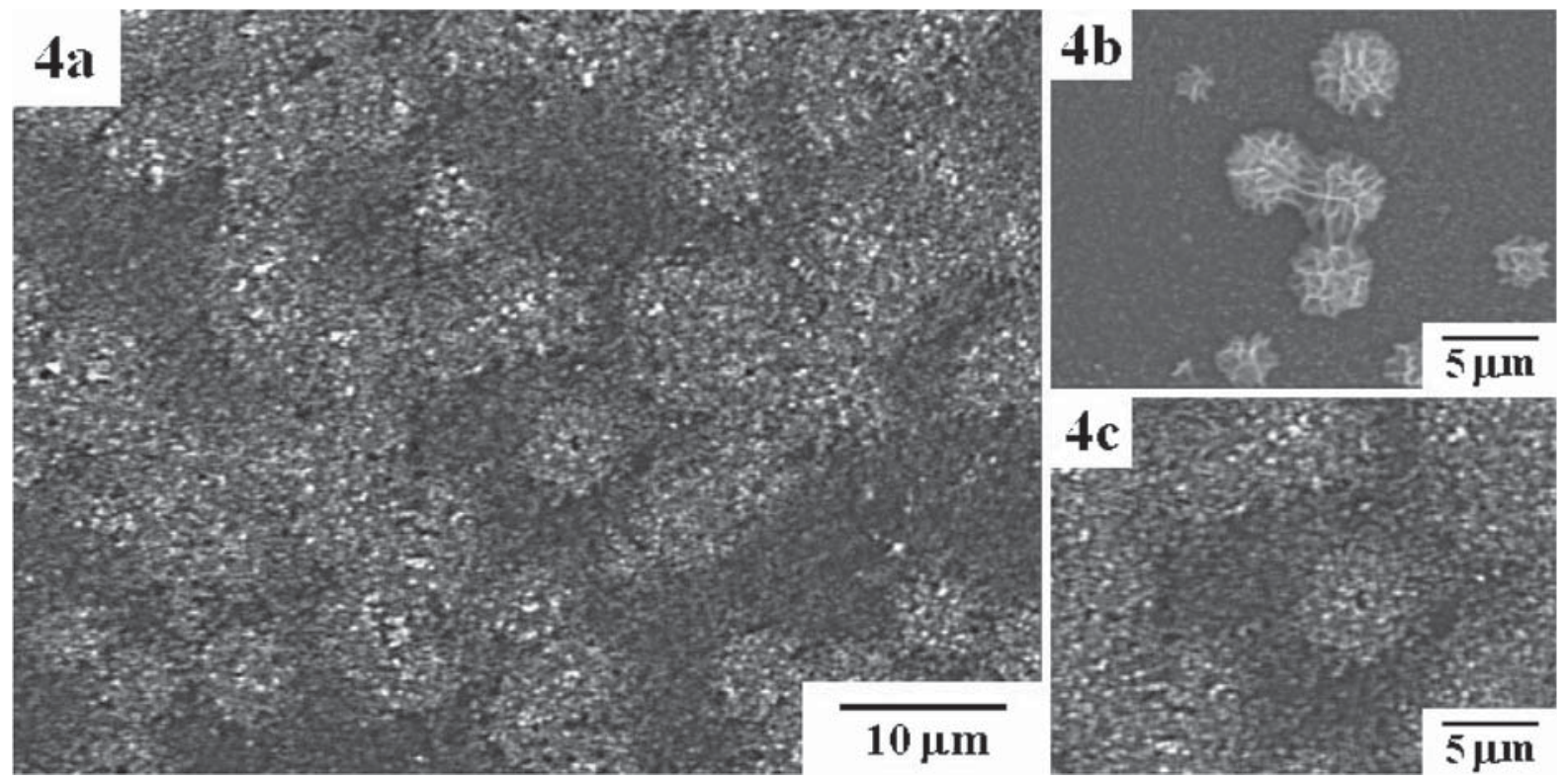

Fig. 4. CuNP formed on the PPy-PSS-MWNT-PEI composite films from hierarchical copper-based micro/nanostructures deposited in oxygen-saturated $0.10 \mathrm{M} \mathrm{CuSO}_{4}$ solutions. a) Large number of the copper nanoparticles formed on composite. The micro/nanostructures after reduction at $-2.00 \mathrm{~V}$ vs. SCE for b) $5 \mathrm{~min}$, and c) $10 \mathrm{~min}$.

tion). This specific potential of $-1.20 \mathrm{~V}$ vs. SCE is chosen as the MWNT-PEI modified film has a characteristic current plateau at this potential, as shown in the next section. Obviously, to obtain results that can be compared quantitatively, the response of the two electrodes was measured at the same potential. The resulting calibration curve, shown in Figure 5a Inset, is linear up to $2 \mathrm{mM}$, at which point the current starts levelling off. by linear regression. The $R^{2}$ is 0.996 , indicating that the experimental response is reasonably linear in this range. The sensitivity of the PPy-PSS-CuNP film was computed as $99.0 \mu \mathrm{A} /\left(\mathrm{mM} \cdot \mathrm{cm}^{2}\right)$. The intercept, i.e., the extrapolated reading of current at zero concentration, is $-8.5 \mu \mathrm{A} / \mathrm{cm}^{2}$, which indicates a residual signal even after background subtraction. This has direct implications on the limit of detection $(L O D)$ of the sensor, i.e., the smallest amount 

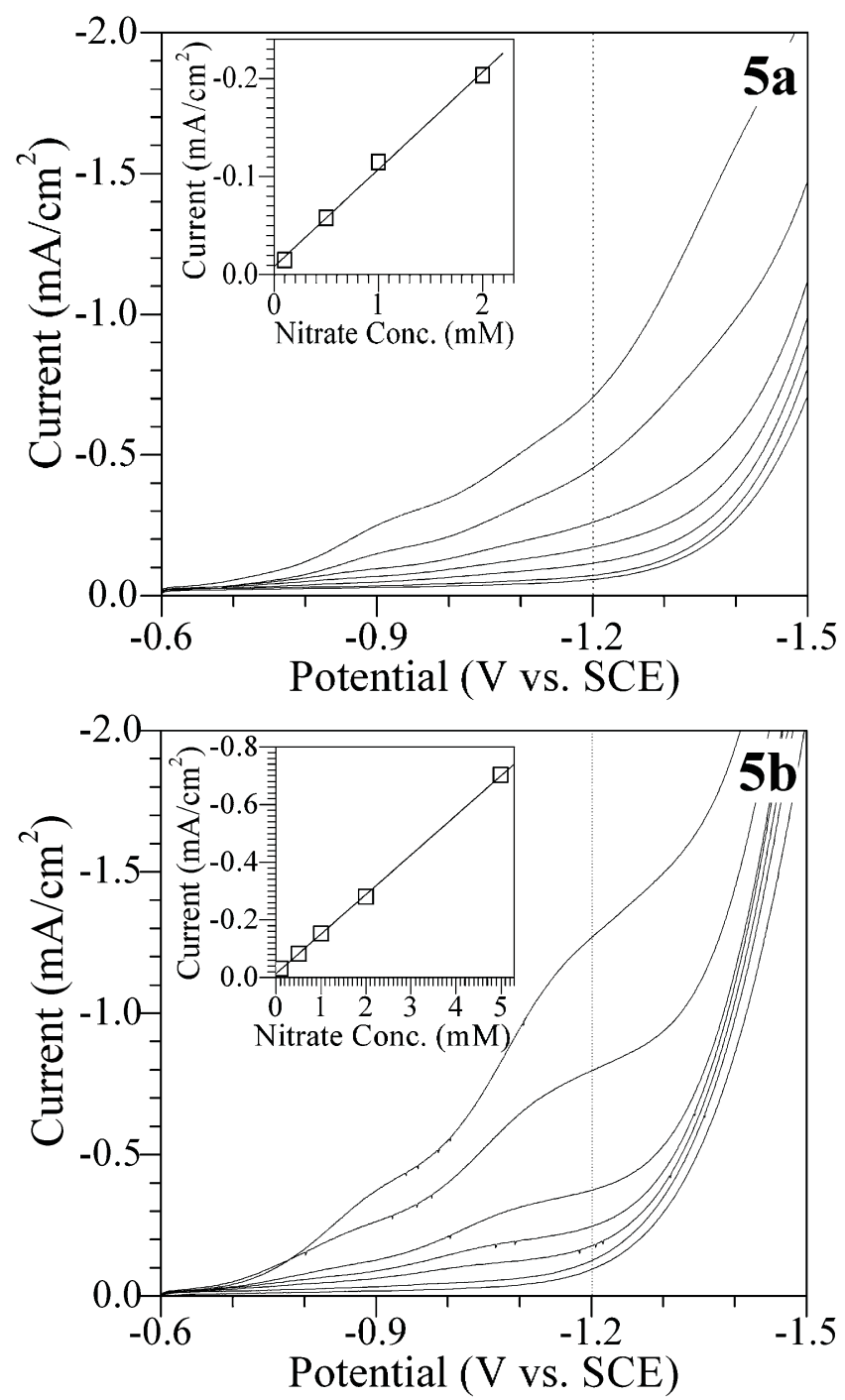

Fig. 5. Voltammetric response of PPy-PSS-CuNP (a) and PPyPSS-MWNT-PEI-CuNP (b) modified electrodes to nitrate ion. The concentration of nitrate is $0.0,0.1,0.5,1.0,2.0,5.0$ and $10.0 \mathrm{mM}$ from the bottom to the top curve. Insets: linear plots of the current registered at $-1.20 \mathrm{~V}$ vs. SCE against the concentration of nitrate. In each case the copper hierarchical structures were deposited on the polymer surface until a charge of $119.4 \mathrm{mC} / \mathrm{cm}^{2}$ was reached.

of nitrate detectable by the electrode [36]. The lowest concentration significantly different from the intercept is $0.5 \mathrm{mM}$ which can be considered the measured $L O D$ for the PPy-PSS-CuNP modified electrode under the related experimental conditions.

\subsubsection{PPy-PSS-MWNT-PEI-CuNP Electrode}

The nitrate sensing performance of a PPy-PSS-MWNTPEI-CuNP electrode was tested using cyclic voltammetry and constant potential amperometry at a RDE.

The voltammetric data are presented Figure $5 \mathrm{~b}$. In the response profiles there are two clear peaks at about $-0.90 \mathrm{~V}$ and $-1.10 \mathrm{~V}$ vs. SCE, also there is evidence of a plateau-like current between $-1.10 \mathrm{~V}$ and $-1.30 \mathrm{~V}$ vs. SCE. A calibration curve was prepared by plotting the current values recorded at $-1.20 \mathrm{~V}$ vs. SCE, against the nitrate concentration (with the previous subtraction of the background current registered without nitrate in solution). The current response for the PPy-PSS-MWNT-PEICuNP electrode is higher, compared to that observed for the film without CNT, and the linear range is wider. The $R^{2}$ is 0.999 , showing the better linearity of the response of the MWNT-PEI modified film compared to the film without nanotubes. The sensitivity of the electrode is also better, $137.2 \mu \mathrm{A} /\left(\mathrm{mM} \cdot \mathrm{cm}^{2}\right)$. In this case, the extrapolated reading of current at zero concentration is slightly higher in the plot, $-13.8 \mu \mathrm{A} / \mathrm{cm}^{2}$, yet the measured $L O D$ of the CNT-modified electrode is $0.1 \mathrm{mM}$ using cyclic voltammetry, five times better than the film without nanotubes.

The substantial improvements obtained with the use of the MWNT-PEI were followed up with a further investigation of the electrode performance by constant potential amperometry at a RDE. It is known that the RDE can improve the mass transport of the analyte from the solution to the electrode surface allowing better $L O D$. In this case the measurements were taken at constant potential, $-1.20 \mathrm{~V}$ vs. SCE, and the current was recorded while various aliquots of nitrate ions were added to the solution. The modified electrode was spun in $0.10 \mathrm{M} \mathrm{Na}_{2} \mathrm{SO}_{4}$ at a constant rotational speed of $2000 \mathrm{rpm}$ during the addition. The amperometric response is characterized by a series of current steps, where each step height is proportional to the added nitrate, as shown in Figure 6a. A large portion of instrumental noise was recorded prior to the start of the nitrate addition while the electrode was polarized in solution without the presence of any nitrate. The distribution of the noise amplitude is in agreement with a normal distribution $\left(R^{2}=0.949, \sigma=4.23 \mu \mathrm{A} / \mathrm{cm}^{2}\right)$. Hence, a current reading has to be higher than $3 \sigma$ in order to be considered different from the noise, i.e., at least more than three times the standard deviation of the noise, $12.69 \mu \mathrm{A} / \mathrm{cm}^{2}$, in absolute value. In order to decrease the noise, the curve was smoothed by 20-point adjacent averaging and the resulting curve is shown in Figure 6b. A general feature of the curve is that within each series of the three additions of nitrate, the current steps decrease. This decrease may be attributed to poisoning effects due to the progressive addition of nitrate to the solution. The detrimental effect of poisoning on the catalytic activity of copper is a well-known issue [9]. In particular, the current steps registered for the $1 \mu \mathrm{M}$ nitrate additions decay rapidly towards zero. For this reason, these additions are not considered further in this discussion. The plots of the current responses against the nitrate concentration are reported in Figure 6b Inset. The responses are collected in three groups corresponding to the order of addition within the same aliquot, i.e., $1^{\text {st }}, 2^{\text {nd }}$ and $3^{\text {rd }}$ additions. On comparing the $1^{\text {st }}, 2^{\text {nd }}$ and $3^{\text {rd }}$ additions at the higher concentrations, it is clear that the overall magnitude of the current response decreases with increasing addition. Instead, the responses in the lower concentration range, 
$5-30 \mu \mathrm{M}$, are randomly distributed. For this reason, it is reasonable to take their average values and standard deviations to perform the linear regression reported in Figure 6c. The $R^{2}$ is 0.992 , indicating some deviation from linearity. The sensitivity of the electrode was computed as $0.64 \mu \mathrm{A} /\left(\mu \mathrm{M} \cdot \mathrm{cm}^{2}\right)$. The extrapolated reading of current at zero concentration is $-3.03 \mu \mathrm{A} / \mathrm{cm}^{2}$ and its $L L_{95}$ is $-5.11 \mu \mathrm{A} / \mathrm{cm}^{2}$. The readings that fall below the $L L_{95}$ in the plot are likely to correspond to the zero concentration and have only a $5 \%$ probability of being related to the nitrate [36]. Following on from this consideration, the current reading at the $5 \mu \mathrm{M}$ nitrate concentration is slightly higher than the $L L_{95}$, however in the plot the lower side of its normal distribution overlaps with that of the $L L_{95}$. As a result, this current reading is not significantly different from the reading at zero concentration. Hence, it cannot be related to the response of the electrode to the addition of nitrate. The current reading at $15 \mu \mathrm{M}$ nitrate concentration, $-13.56 \mu \mathrm{A} / \mathrm{cm}^{2}$, is well above the $L L_{95}$, however it is very near to the detection limit enforced by the noise, $-12.69 \mu \mathrm{A} / \mathrm{cm}^{2}$. Following the same reasoning as before, the limiting value of this experimental reading, $-13.56+2 \sigma=-11.14 \mu \mathrm{A} / \mathrm{cm}^{2}$, is lower in the plot than the detection limit due to the noise, hence it must be rejected since there is a finite probability that this signal is due to noise. Accordingly, the measured $L O D$ of the PPy-PSSMWNT-PEI-CuNP electrode is $30 \mu \mathrm{M}$ nitrate which is significantly above the $3 \sigma$ of the noise. It is easy to see that the LOD of the modified electrode is affected by the noise. Further optimization of the electrode and the employment of techniques to reduce the noise level could possibly enhance the performance of the sensor bringing it closer to the best values reported in the literature for other sensors, $0.1-10 \mu \mathrm{M}$ [9]. The sensor-to-sensor reproducibility was investigated at a nitrate concentration of $100 \mu \mathrm{M}$. Three independently made sensors showed a reasonable reproducibility with an average $R S D$ of $7 \%(n=$ 6). However, poisoning effects on the CuNP remain an issue affecting the repeatability and stability of the sensor.

\subsection{Electrochemical Impedance of PPy-PSS and PPy- PSS-MWNT-PEI Films}

The nitrate sensing performance of the electrodes modified with CuNP was significantly improved by introducing MWNT-PEI in the PPy-PSS films. In order to evaluate the effect of the MWNT-PEI, the electrical properties of the PPy-PSS films, with and without CNT, were investigated using electrochemical impedance spectroscopy (EIS).

PPy-PSS films have been the object of many EIS characterization studies. Ren and Pickup [37,38] carried out detailed studies of both ion and electron transport in such films. In their works, they used the dual rail transmission line circuit originally proposed by Albery et al. $[39,40]$. In this circuit two film resistances, one ionic, $R_{\text {ion }}$, and one electronic, $R_{\mathrm{e}}$, are connected in parallel through a group
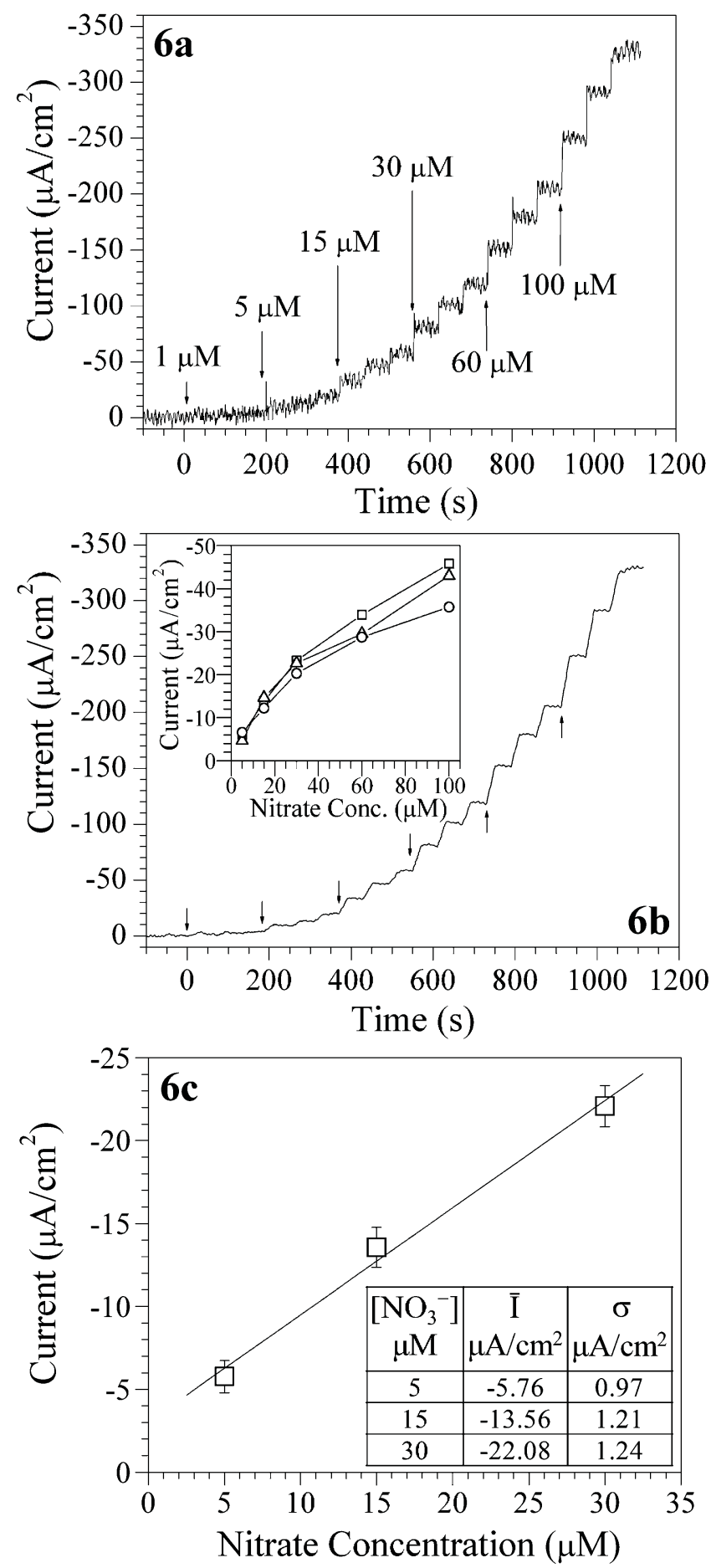

Fig. 6. Amperometric response of PPy-PSS-MWNT-PEI-CuNP film modified RDE. a) Raw data and amount of nitrate added three times in series for each aliquot. b) 20-point adjacent averaging. Inset: Current of the $1^{\text {st }}(\square), 2^{\text {nd }}(\triangle)$ and $3^{\text {rd }}(\bigcirc)$ additions for each aliquot. c) Linear regression and values of the averaged aliquots 5,15 and $30 \mu \mathrm{M}$.

of capacitors representing the pseudocapacitance of the film, $C_{\mathrm{F}}$. They found that above $-0.60 \mathrm{~V}$ vs. $\mathrm{Ag} / \mathrm{AgCl}$ the impedance response of the film is described by a single transmission line, $R_{\text {ion }}$, since at this potential $R_{\mathrm{e}}$ is negligible. Below this potential, $R_{\mathrm{e}}$ is comparable to $R_{\text {ion }}$. At 
more negative potentials, such as below $-0.75 \mathrm{~V}$ vs. $\mathrm{Ag} /$ $\mathrm{AgCl}$, the $R_{\mathrm{e}}$ term dominates over $R_{\text {ion }}$. In this case, the film impedance is reasonably described by a single transmission line governed by $R_{\mathrm{e}}$. This transition is related to the electrochemistry of the polymer film. In fact, in its oxidized state (low $R_{\mathrm{e}}$ ) the film is deprived of mobile counterions (high $R_{\text {ion }}$ ) since the negative charge of the dopant, PSS, is counter-balanced by the positive charge of oxidized PPy. Conversely, in its reduced state (high $R_{e}$ ) the film is rich in mobile counterions (low $R_{\text {ion }}$ ) necessary to maintain the charge balance. Komura et al. [41] came to the same conclusions that the reduced film has high ionic conductivity due to the incorporation of cations, while the electronic resistance increases with decreasing oxidation level. The present characterization was performed to obtain the experimental data necessary for a direct comparison between PPy-PPS and PPy-PSSMWNT-PEI films. The experimental conditions used in the present work are different from those of the published studies. In this study, the PPy films are thinner, $0.12 \mu \mathrm{m}$ compared to $2 \mu \mathrm{m}$, and the electrolyte is also different, $0.10 \mathrm{M} \mathrm{Na}_{2} \mathrm{SO}_{4}$ instead of $0.2 \mathrm{M} \mathrm{NaClO}_{4}$ or $0.5 \mathrm{M}$ $\mathrm{NaCl}$. These differences can affect the impedance response of the films. In fact, thinner films are characterized by a higher molecular order compared to thicker films [42]. Also, sulfate ions can be exchanged by the PPy-PSS film when cycled in sulfate electrolytes [43]. These ions can act as electrostatic cross-linking points between oxidized moieties of the polymer.

The impedance responses of PPy-PSS and PPy-PSSMWNT-PEI films at $-1.00 \mathrm{~V}$ vs. SCE in $0.10 \mathrm{M} \mathrm{Na}_{2} \mathrm{SO}_{4}$ with their corresponding equivalent circuits and fitting results are reported in Figures 7 and 8, respectively.

The PPy-PSS film is completely reduced at $-1.00 \mathrm{~V}$ vs. SCE and the equivalent circuit is composed of five elements (Figure 7). The solution resistance $\left(R_{\mathrm{s}}\right)$, a constant phase element $\left(\mathrm{CPE}_{\text {int }}\right)$ in parallel to a resistance $\left(R_{\text {int }}\right)$, and another constant phase element $\left(\mathrm{CPE}_{\mathrm{i}}\right)$, in parallel to a resistance $\left(R_{\mathrm{e}}\right)$. At this potential the negative charge of the dopant is neutralized by insertion of sodium ions to give $\mathrm{PPy}^{0} \mathrm{PSS}^{-} \mathrm{Na}^{+}$. The ionic resistance, $R_{\text {ion }}$, of the film is negligible compared to the electronic resistance, $R_{e}$ [38]. The electronic resistance, $R_{\mathrm{e}}$, is about $40 \mathrm{k} \Omega$. This is consistent with the reduction of the film to its insulating state. The $\mathrm{CPE}_{\mathrm{i}}(n=0.57)$, in parallel to $R_{\mathrm{e}}$, is due to the diffusion of $\mathrm{Na}^{+}$ions inside the reduced film, in fact it represents a slightly distorted Warburg element. The $\mathrm{CPE}_{\text {int }}$ and $R_{\text {int }}$ are related to the capacitance and electron transfer resistance at the electrode/film interface as observed and supported by the impedance analysis of both Ren and Komura [38,41]. Komura et al. [41] found that the $R_{\text {int }}$ at the metal/film interface was emerging as a result of the decreasing concentration of hole carriers in the PPy-PSS film.

There is no previous impedance characterization of PPy-PSS-MWNT-PEI films, since these nanocomposites are presented in this work for the first time. However, different studies on the properties of composites made from PPy and CNT are available. Chen et al. [32] used carboxyl-functionalized CNT directly as a dopant for the electrochemical polymerization of Py. In this case, the impedance spectra of the composite films were recorded at $0.20 \mathrm{~V},-0.20 \mathrm{~V}$ and $-0.60 \mathrm{~V}$ vs. SCE. The result was a response characterized only by diffusive and capacitive components regardless of the oxidation state of the films. Such behaviour was entirely new as reduced PPy films are insulating. The CNT were responsible for this conductivity in the composite films even when PPy was in its reduced state. Wu and Lin [44] measured conductivities two orders of magnitude higher for PPy modified with MWNT compared to their plain counterparts. The overall effect of the addition of MWNT to the PPy is then to improve the conductivity and capacitance of the films. This is also observed in the present study. The impedance response of PPy-PSS-MWNT-PEI at $-1.00 \mathrm{~V}$ vs. SCE is given in Figure 8.

The equivalent circuit is composed of a solution resistance $\left(\mathrm{R}_{\mathrm{s}}\right)$, a distorted film capacitance $\left(\mathrm{CPE}_{\mathrm{f}}, n=0.91\right)$, and a distorted Warburg element $\left(\mathrm{CPE}_{\mathrm{i}}, n=0.4\right)$. Another
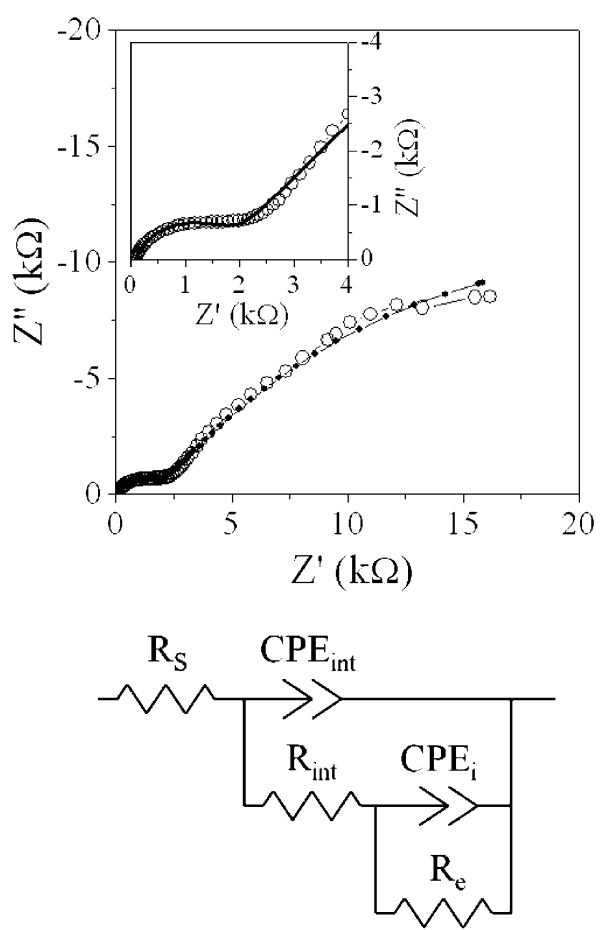

$$
\begin{aligned}
& \mathrm{R}_{\mathrm{s}}=96.2 \Omega \\
& \mathrm{R}_{\text {int }}=1823 \Omega \\
& \mathrm{R}_{\mathrm{e}}=40830 \Omega \\
& \mathrm{CPE}_{\text {int }}: A=1.5 \times 10^{-5} \Omega^{-1} \mathrm{~s}^{0.77}, n=0.77 \\
& \mathrm{CPE}_{\mathrm{i}}: A=5.1 \times 10^{-4} \Omega^{-1} \mathrm{~s}^{0.57}, n=0.57
\end{aligned}
$$

Fig. 7. Raw $(\circ)$ and simulated $(\bullet)$ data, equivalent circuit and fitting results of the impedance response of a PPy-PSS film recorded at $-1.00 \mathrm{~V}$ vs. SCE in $0.10 \mathrm{M} \mathrm{Na}_{2} \mathrm{SO}_{4}$. Frequency range $2 \times 10^{-3}-65 \times 10^{3} \mathrm{~Hz}, \mathrm{AC}$ amplitude $5 \mathrm{mV}$, equilibration time at bias voltage $30 \mathrm{~min}$. Fitting software Z-View 2.3. 

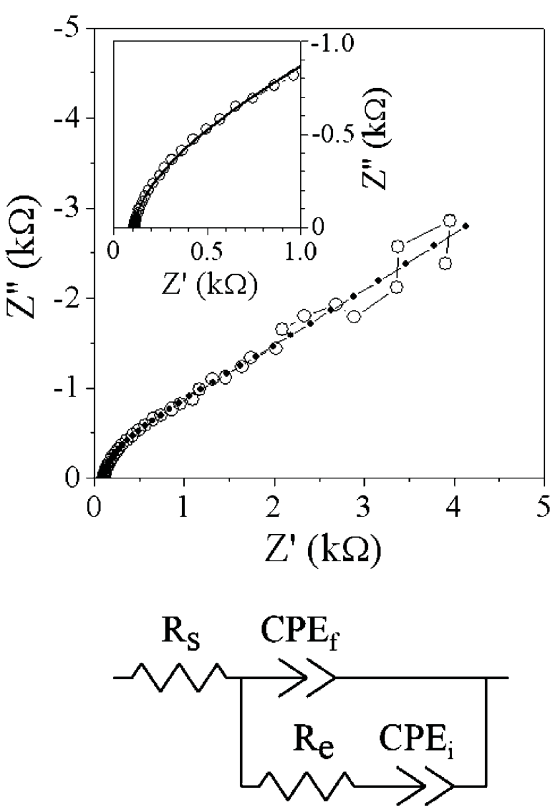

$\mathrm{R}_{\mathrm{S}}=108.0 \Omega$

$\mathrm{R}_{\mathrm{e}}=523.9 \Omega$

$$
\mathrm{CPE}_{\mathrm{f}}: A=1.8 \times 10^{-4} \Omega^{-1} \mathrm{~s}^{0.91}, n=0.91
$$$$
\mathrm{CPE}_{\mathrm{i}}: A=6.4 \times 10^{-4} \Omega^{-1} \mathrm{~S}^{0.4}, n=0.4
$$

Fig. 8. Raw $(\circ)$ and simulated $(\bullet)$ data, equivalent circuit and fitting results of the impedance response of a PPy-PSS-MWNTPEI film recorded at $-1.00 \mathrm{~V}$ vs. SCE in $0.10 \mathrm{M} \mathrm{Na}_{2} \mathrm{SO}_{4}$. Frequency range $1 \times 10^{-2}-65 \times 10^{3} \mathrm{~Hz}$, AC amplitude $5 \mathrm{mV}$, equilibration time at bias voltage $30 \mathrm{~min}$. Fitting software Z-View 2.3.

element is also present, the electronic resistance $R_{e}$, of the reduced PPy-PSS-MWNT-PEI film. According to the expected response of the composite, the capacitance of the reduced film is about $27 \mathrm{~F} / \mathrm{g}$. The diffusion of $\mathrm{Na}^{+}$ ions into the film, which enter to counterbalance the negative charge of the dopant, is described by the Warburg element. The key element of this equivalent circuit is the electronic resistance, $\mathrm{R}_{\mathrm{e}}$, which appeares once the PPy-PSS-MWNT-PEI film is completely reduced. This is in accordance with the predominant resistive contribution of $\mathrm{R}_{\mathrm{e}}$ observed also for the plain PPy-PSS films. However, this time the resistance is considerably lower, $524 \Omega$ compared with $40830 \Omega$. This higher conductivity is consistent with the presence of the MWNT-PEI which allows more efficient electron transport through the reduced film [44].

The nitrate sensing performance of the CuNP modified electrodes are greatly improved by the incorporation of MWNT-PEI in the PPy-PSS films. The sensitivity, linear range of the response and the LOD are all enhanced by the presence of the CNT. The EIS characterization shows clearly that the reduced PPy-PSS film, which is an electrical insulator, becomes a conductor due to the addition of the modified CNT. The improvement of electrical conductivity is the major factor for the enhanced nitrate sensing performance in the presence of MWNT-PEI.

\section{Conclusions}

Copper-based hierarchical micro/nanostructures were reduced to metallic copper nanoparticles. This transition was documented by SEM imaging. The electrode modified with the copper nanoparticles was used to sense nitrate ions both voltammetrically and amperometrically. The sensing performance was enhanced by introducing polyethyleneimine-modified multiwall carbon nanotubes in the polymer film. An electrochemical impedance study showed that the nanotubes had significantly enhanced the conductivity of the films, particularly at potentials where the films were reduced. In relation to the performance in the sensing of nitrates, the PPy-PSS-CuNP films had a sensitivity of $99.0 \mu \mathrm{A} /\left(\mathrm{mM} \cdot \mathrm{cm}^{2}\right)$, a linear range from $0.5-$ $2.0 \mathrm{mM}$, and a measured voltammetric detection limit was $0.5 \mathrm{mM}$. The PPy-PSS-MWNT-PEI-CuNP had a sensitivity of $137.2 \mu \mathrm{A} /\left(\mathrm{mM} \cdot \mathrm{cm}^{2}\right)$, the linear range was between 0.1-5.0 $\mathrm{mM}$, and the measured voltammetric detection limit was $0.1 \mathrm{mM}$. This detection limit was $30 \mu \mathrm{M}$ using constant potential amperometry at a rotating disk electrode. Studies are required to investigate the selectivity and stability of these materials with respect to their application as sensors.

\section{Acknowledgements}

This work was supported by the Environmental Protection Agency Ireland as part of the Science, Technology, Research and Innovation for the Environment Programme financed by the Irish Government. Additional funding was provided by the Science Foundation Ireland through the National Access Programme of the Tyndall National Institute, University College Cork, and also the Research Frontiers Programme 08/RFP/MTR1261.

\section{References}

[1] F. W. Campbell, R. G. Compton, Anal. Bioanal. Chem. 2010, 396, 241.

[2] S. Roth, S. Blumentritt, M. Burghard, E. Cammi, D. Carrol, S. Curran, G. Düsberg, K. Liu, J. Muster, G. Philipp, T. Rabenau, Synth. Met. 1998, 94, 105.

[3] A. Aviram, M. A. Ratner, Chem. Phys. Lett. 1974, 29, 277.

[4] A. Z. Moshfegh, J. Phys. D, Appl. Phys. 2009, 42, 233001.

[5] C. W. Welch, R. G. Compton, Anal. Bioanal. Chem. 2006, $384,601$.

[6] C. M. Welch, M. E. Hyde, C. E. Banks, R. G. Compton, Anal. Sci. 2005, 21, 1421.

[7] F. R. Greer, M. Shannon, Pediatrics 2005, 116, 784.

[8] V. H. Smith, G. D. Tilman, J. C. Nekola, Environ. Pollut. 1999, 100, 179 .

[9] M. J. Moorcroft, J. Davis, R. G. Compton, Talanta 2001, 54, 785 .

[10] S. Ward-Jones, C. E. Banks, A. O. Simm, L. Jiang, R. G. Compton, Electroanalysis 2005, 17, 1806.

[11] J. Davis, M. J. Moorcroft, S. J. Wilkins, R. G. Compton, M. F. Cardosi, Analyst 2000, 125, 737. 
[12] European Council Directive 98/83/EC; The Quality of Water Intended for Human Consumption 1998.

[13] United States Environmental Protection Agency EPA 816F-09-004; National Primary Drinking Water Regulations 2006.

[14] X. J. Zhou, A. J. Harmer, N. F. Heinig, K. T. Leung, Langmuir 2004, 20, 5109.

[15] D. K. Sarkar, X. J. Zhou, A. Tannous, M. Louie, K. T. Leung, Solid State Commun. 2003, 125, 365.

[16] D. K. Sarkar, X. J. Zhou, A. Tannous, K. T. Leung, J. Phys. Chem. 2003, 107, 2879.

[17] V. Tsakova, J. Solid State Electrochem. 2008, 12, 1421.

[18] J. N. Coleman, S. Curran, A. B. Dalton, A. P. Davey, B. McCarthy, W. Blau, R. C. Barklie, Phys. Rev. B 1998, 58, 7492.

[19] J. N. Coleman, S. Curran, A. B. Dalton, A. P. Davey, B. McCarthy, W. Blau, R. C. Barklie, Synth. Met. 1999, 102, 1174.

[20] Y. P. Sun, K. F. Fu, Y. Lin, W. J. Huang. Accounts Chem. Res. 2002, 35, 1096.

[21] M. S. P. Shaffer, X. Fan, A. H. Windle, Carbon 1998, 36, 1603.

[22] K. S. Liao, J. A. Talla, S. D. Yambem, D. Birx, G. Chen, F. Coldren, D. L. Carroll, L. J. Ci, P. M. Ajayan, D. H. Zhang, S. A. Curran, J. Mater. Res. 2010, 25, 1741.

[23] S. A. Curran, D. H. Zhang, W. T. Wondmagegn, A. V. Ellis, J. Cech, D. S. Roth, D. L. Carroll, J. Mater. Res. 2006, 21, 1071.

[24] S. A. Curran, J. Talla, S. Dias, D. H. Zhang, D. Carroll, D. Birx, J. Appl. Phys. 2009, 105, 073711.

[25] E. Andreoli, D. A. Rooney, W. Redignton, R. Gunning, C. B. Breslin, J. Phys. Chem. C 2011, 115, 8725.

[26] K. S. Liao, A. Wan, J. D. Batteas, D. E. Bergbreiter, Langmuir 2008, 24, 4245.

[27] A. L. Beilby, T. A. Sasaki, H. M. Stern, Anal. Chem. 1995, 67, 976.
[28] T. Nagaoka, T. Yoshino, Anal. Chem. 1986, 58, 1037.

[29] R. C. Engstrom, Anal. Chem. 1982, 54, 2310.

[30] M. Hughes, G. Z. Chen, M. S. P. Shaffer, D. J. Fray, A. H. Windle, Chem. Mater. 2002, 14, 1610.

[31] M. Hughes, M. S. P. Shaffer, A. C. Renouf, C. Singh, G. Z. Chen, J. Fray, A. H. Windle, Adv. Mater. 2002, 14, 382.

[32] G. Z. Chen, M. S. P. Shaffer, D. Coleby, G. Dixon, W. Z Zhou, D. J. Fray, A. H. Windle, Adv. Mater. 2000, 12, 522.

[33] R. K. Sharma, A. Karakoti, S. Seal, L. Zhai, J. Power Sources 2010, 195, 1256.

[34] G. E. Dima, A. C. A. de Vooys, M. T. M. Koper, J. Electroanal. Chem. 2003, 554, 15.

[35] N. Aouina, H. Cachet, C. Debiemme-chouvy, T. M. T. Thi, Electrochim. Acta 2010, 55, 7341.

[36] A. Hubaux, G. Vos, Anal. Chem. 1970, 42, 849.

[37] X. M. Ren, P. G. Pickup, J. Phys. Chem. 1993, 97, 5356.

[38] X. M. Ren, P. G. Pickup, J. Electroanal. Chem. 1997, 420, 251.

[39] W. J. Albery, C. M. Elliott, A. R. Mount, J. Electroanal. Chem. 1990, 288, 15.

[40] W. J. Albery, Z. Chen, B. R. Horrocks, A. R. Mount, P. J. Wilson, D. Bloor, A. T. Monkman, C. M. Elliott, Faraday Discuss. 1989, 88, 247.

[41] T. Komura, S. Goisihara, T. Yamaguti, K. Takahasi, J. Electroanal. Chem. 1998, 456, 121.

[42] R. Yang, K. Naoi, D. F. Evans, W. H. Smyrl, W. A. Hendrickson, Langmuir 1991, 7, 556.

[43] F. B. Li, W. J. Albery, J. Chem. Soc.-Faraday Trans. 1991, 87, 2949.

[44] T. M. Wu, S. H. Lin, J. Pol. Sci. Part A-Pol. Chem. 2006, 44, 6449. 